\title{
Maximal operator with rough kernel and its commutators in generalized weighted Morrey spaces
}

\author{
Vugar H. Hamzayev *
}

Received: 08.08.2019 / Revised: 28.02.2020 / Accepted: 10.04.2020

\begin{abstract}
Let $\Omega \in L_{q}\left(S^{n-1}\right)$ be a homogeneous function of degree zero with $q>1$. In this paper, we study the boundedness of the maximal operator with rough kernels $M_{\Omega}$ and its commutators $\left[b, M_{\Omega}\right]$ on generalized weighted Morrey spaces $M_{p, \varphi}(w)$. We find the sufficient conditions on the pair $\left(\varphi_{1}, \varphi_{2}\right)$ with $q^{\prime} \leq p<\infty, p \neq 1$ and $w \in A_{p / q^{\prime}}$ or $1<p \leq q$ and $w^{1-p^{\prime}} \in A_{p^{\prime}} / q^{\prime}$ which ensures the boundedness of the operators $M_{\Omega}$ from one generalized weighted Morrey space $M_{p, \varphi_{1}}(w)$ to another $M_{p, \varphi_{2}}(w)$ for $1<p<\infty$. We find the sufficient conditions on the pair $\left(\varphi_{1}, \varphi_{2}\right)$ with $b \in B M O\left(\mathbb{R}^{n}\right)$ and $q^{\prime} \leq p<\infty$, $p \neq 1, w \in A_{p / q^{\prime}}$ or $1<p \leq q, w^{1-p^{\prime}} \in A_{p^{\prime}} / q^{\prime}$ which ensures the boundedness of the operators $\left[b, M_{\Omega}\right]$ from $M_{p, \varphi_{1}}(w)$ to $M_{p, \varphi_{2}}(w)$ for $1<p<\infty$. In all cases the conditions for the boundedness of the operators $T_{\Omega},\left[b, T_{\Omega}\right]$ are given in terms of supremal-type inequalities on $\left(\varphi_{1}, \varphi_{2}\right)$ and $w$, which do not assume any assumption on monotonicity of $\varphi_{1}(x, r), \varphi_{2}(x, r)$ in $r$.
\end{abstract}

Keywords. Maximal operator; rough kernel; generalized weighted Morrey spaces; commutator; $A_{p}$ weights

Mathematics Subject Classification (2010): 42B25, 42B35

\section{Introduction}

It is well-known that the commutator is an important integral operator and it plays a key role in harmonic analysis. In 1965, Calderon [6,7] studied a kind of commutators, appearing in Cauchy integral problems of Lip-line. Let $K$ be a Calderón-Zygmund singular integral operator and $b \in B M O\left(\mathbb{R}^{n}\right)$. A well known result of Coifman, Rochberg and Weiss [8] states that the commutator operator $[b, K] f=K(b f)-b K f$ is bounded on $L_{p}\left(\mathbb{R}^{n}\right)$ for $1<p<\infty$. The commutator of Calderón-Zygmund operators plays an important role in studying the regularity of solutions of elliptic partial differential equations of second order (see, for example, [9-11,15,24,26]).

The classical Morrey spaces were originally introduced by Morrey in [34] to study the local behavior of solutions to second order elliptic partial differential equations. For the properties and applications of classical Morrey spaces, we refer the readers to $[9,10,12$, 15,19]. Guliyev, Mizuhara and Nakai $[17,33,38]$ introduced generalized Morrey spaces

\footnotetext{
* Corresponding author
}

V.H. Hamzayev

Nakhchivan Teacher-Training Institute, Nakhchivan, Azerbaijan

Institute of Mathematics and Mechanics of NAS of Azerbaijan, Baku, Azerbaijan

E-mail: vugarhamzayev@yahoo.com 
$M^{p, \varphi}\left(\mathbb{R}^{n}\right)$ (see, also [18, 19,21,39]). Recently, Komori and Shirai [31] considered the weighted Morrey spaces $L^{p, \kappa}(w)$ and studied the boundedness of some classical operators such as the Hardy-Littlewood maximal operator, the Calderón-Zygmund operator on these spaces. Guliyev [20] gave a concept of generalized weighted Morrey space $M_{p, \varphi}(w)$ which could be viewed as extension of both generalized Morrey space $M_{p, \varphi}$ and weighted Morrey space $L^{p, \kappa}(w)$. In [20] Guliyev also studied the boundedness of the classical operators and its commutators in these spaces $M_{p, \varphi}(w)$, see also Guliyev et al. [3,11,13,22,25, 26, 28, 29].

Watson [40] and independently by Duoandikoetxea [14] established weighted $L_{p}$ boundedness for the singular integral operators with rough kernels and their commutators.

Let $S^{n-1}=\left\{x \in \mathbb{R}^{n}:|x|=1\right\}$ the unit sphere of $\mathbb{R}^{n}(n \geq 2)$ equipped with the normalized Lebesgue measure $d \sigma=d \sigma\left(x^{\prime}\right)$.

Suppose that $\Omega$ satisfies the following conditions.

(i) $\Omega$ is a homogeneous function of degree zero on $\mathbb{R}^{n}$. That is,

$$
\Omega(t x)=\Omega(x)
$$

for all $t>0$ and $x \in \mathbb{R}^{n}$.

Let $f \in L_{1}^{\text {loc }}\left(\mathbb{R}^{n}\right)$. The maximal operator with rough kernel $M_{\Omega}$ is defined by

$$
M_{\Omega} f(x)=\sup _{t>0}|B(x, t)|^{-1} \int_{B(x, t)}|\Omega(x-y)||f(y)| d y .
$$

The commutators generated by a suitable function $b$ and the operator $M_{\Omega}$ is formally defined by

$$
\left[b, M_{\Omega}\right] f=M_{\Omega}(b f)-b M_{\Omega}(f) .
$$

It is obvious that when $\Omega \equiv 1, M_{\Omega}$ is the Hardy-Littlewood maximal operator $M$. For $b \in L_{1}^{\text {loc }}\left(\mathbb{R}^{n}\right)$ the commutator of the maximal operator $M_{\Omega, b}$ is defined by

$$
M_{\Omega, b} f(x)=\sup _{t>0}|B(x, t)|^{-1} \int_{B(x, t)}|\Omega(x-y)||b(x)-b(y)||f(y)| d y .
$$

Therefore, it will be an interesting thing to study the property of $M_{\Omega}$. The main purpose of this paper is to show that maximal operator with rough kernels $M_{\Omega}$ is bounded from one generalized weighted Morrey space $M_{p, \varphi_{1}}(w)$ to another $M_{p, \varphi_{2}}(w), 1<p<\infty$. We find the sufficient conditions on the pair $\left(\varphi_{1}, \varphi_{2}\right)$ with $b \in B M O\left(\mathbb{R}^{n}\right)$ and $q^{\prime} \leq p<\infty, p \neq 1$, $w \in A_{p / q^{\prime}}$ or $1<p \leq q, w^{1-p^{\prime}} \in A_{p^{\prime} / q^{\prime}}$ which ensures the boundedness of the commutator operators $\left[b, M_{\Omega}\right]$ from $M_{p, \varphi_{1}}(w)$ to $M_{p, \varphi_{2}}(w)$ for $1<p<\infty$.

By $A \lesssim B$ we mean that $A \leq C B$ with some positive constant $C$ independent of appropriate quantities. If $A \lesssim B$ and $B \lesssim A$, we write $A \approx B$ and say that $A$ and $B$ are equivalent.

\section{Preliminaries}

Next we will give the weighted boundedness of maximal operator $M_{\Omega}$ with rough kernel and its commutator. In their proof, the weighted boundedness of the maximal operator $M_{\Omega}$ with rough kernel (for its definition, see (1.2)) is needed, while the latter itself is of great significance.

Theorem 2.1 [14] Suppose that $\Omega$ satisfies the condition (1.1) and $\Omega \in L_{q}\left(S^{n-1}\right), 1<$ $q \leq \infty$. Then for every $q^{\prime} \leq p<\infty, p \neq 1$ and $w \in A_{p / q^{\prime}}$ or $1<p \leq q, p \neq \infty$ and $w^{1-p^{\prime}} \in A_{p^{\prime}} / q^{\prime}$, there is a constant $C$ independent of $f$ such that

$$
\left\|M_{\Omega}(f)\right\|_{L_{p, w}} \leq C\|f\|_{L_{p, w}} .
$$


Theorem 2.2 [4] Suppose that $\Omega$ satisfies the condition (1.1) and $\Omega \in L_{q}\left(S^{n-1}\right), 1<$ $q \leq \infty$. Let also $b \in B M O\left(\mathbb{R}^{n}\right)$. Then for every $q^{\prime} \leq p<\infty, p \neq 1$ and $w \in A_{p / q^{\prime}}$ or $1<p \leq q, p \neq \infty$ and $w^{1-p^{\prime}} \in A_{p^{\prime}} / q^{\prime}$, there is a constant $C$ independent of $f$ such that

$$
\left\|M_{\Omega, b}(f)\right\|_{L_{p, w}} \leq C\|f\|_{L_{p, w}} .
$$

For a function $b$ defined on $\mathbb{R}^{n}$, we denote

$$
b^{-}(x):= \begin{cases}0, & \text { if } b(x) \geq 0 \\ |b(x)|, & \text { if } b(x)<0\end{cases}
$$

and $b^{+}(x):=|b(x)|-b^{-}(x)$. Obviously, $b^{+}(x)-b^{-}(x)=b(x)$.

The following relations between $[b, M]$ and $M_{b}$ are valid:

Let $b$ be any non-negative locally integrable function. Then

$$
|[b, M] f(x)| \leq M_{b}(f)(x), \quad x \in \mathbb{R}^{n}
$$

holds for all $f \in L_{\text {loc }}^{1}\left(\mathbb{R}^{n}\right)$.

If $b$ is any locally integrable function on $\mathbb{R}^{n}$, then

$$
|[b, M] f(x)| \leq M_{b}(f)(x)+2 b^{-}(x) M f(x), \quad x \in \mathbb{R}^{n}
$$

holds for all $f \in L_{\text {loc }}^{1}\left(\mathbb{R}^{n}\right)$ (see, for example, [1]).

In the sequel $\mathfrak{M}\left(\mathbb{R}_{+}\right), \mathfrak{M}^{+}\left(\mathbb{R}_{+}\right)$and $\mathfrak{M}^{+}\left(\mathbb{R}_{+} ; \uparrow\right)$ stand for the set of Lebesgue-measurable functions on $\mathbb{R}_{+}$, and its subspaces of nonnegative and nonnegative non-decreasing functions, respectively. We also denote

$$
\mathbb{A}=\left\{\varphi \in \mathfrak{M}^{+}\left(\mathbb{R}_{+} ; \uparrow\right): \lim _{t \rightarrow 0^{+}} \varphi=0\right\}
$$

Let $u$ be a continuous and non-negative function on $\mathbb{R}_{+}$. We define the supremal operator $\bar{S}_{u}$ by

$$
\left(\bar{S}_{u} g\right)(t):=\|u g\|_{L_{\infty}(t, \infty)}, \quad t \in(0, \infty),
$$

The following theorem was proved in [5].

Theorem 2.3 [5] Suppose that $v_{1}$ and $v_{2}$ are nonnegative measurable functions such that $0<\left\|v_{1}\right\|_{L_{\infty}(0, \cdot)}<\infty$ for every $t>0$. Let $u$ be a continuous nonnegative function on $\mathbb{R}$. Then the operator $\bar{S}_{u}$ is bounded from $L_{\infty, v_{1}}\left(\mathbb{R}_{+}\right)$to $L_{\infty, v_{2}}\left(\mathbb{R}_{+}\right)$on the cone $\mathbb{A}$ if and only if

$$
\left\|v_{2} \bar{S}_{u}\left(\left\|v_{1}\right\|_{L_{\infty}(\cdot, \infty)}^{-1}\right)\right\|_{L_{\infty}\left(\mathbb{R}_{+}\right)}<\infty .
$$




\section{Generalized weighted Morrey spaces}

The classical Morrey spaces $M_{p, \lambda}$ were originally introduced by Morrey in [34] to study the local behavior of solutions to second order elliptic partial differential equations. For the properties and applications of classical Morrey spaces, we refer the readers to $[16,32]$.

We recall that a weight function $w$ is in the Muckenhoupt class $A_{p}[35], 1<p<\infty$, if

$$
\begin{aligned}
{[w]_{A_{p}}: } & =\sup _{B}[w]_{A_{p}(B)} \\
& =\sup _{B}\left(\frac{1}{|B|} \int_{B} w(x) d x\right)\left(\frac{1}{|B|} \int_{B} w(x)^{1-p^{\prime}} d x\right)^{p-1}
\end{aligned}
$$

where the sup is taken with respect to all the balls $B$ and $\frac{1}{p}+\frac{1}{p^{\prime}}=1$. Note that, for all balls $B$ using Hölder's inequality, we have that

$$
[w]_{A_{p}(B)}^{1 / p}=|B|^{-1}\|w\|_{L_{1}(B)}^{1 / p}\left\|w^{-1 / p}\right\|_{L_{p^{\prime}}(B)} \geq 1 .
$$

For $p=1$, the class $A_{1}$ is defined by the condition $M w(x) \leq C w(x)$ with $[w]_{A_{1}}=$ $\sup _{x \in \mathbb{R}^{n}} \frac{M w(x)}{w(x)}$, and for $p=\infty A_{\infty}=\bigcup_{1 \leq p<\infty} A_{p}$ and $[w]_{A_{\infty}}=\inf _{1 \leq p<\infty}[w]_{A_{p}}$.

Remark 3.1 It is known that

$$
w^{1-p^{\prime}} \in A_{p^{\prime} / q^{\prime}} \Rightarrow\left[w^{1-p^{\prime}}\right]_{A_{p^{\prime} / q^{\prime}}^{q^{\prime} / p^{\prime}}}=|B|^{-1}\left\|w^{1-p^{\prime}}\right\|_{L_{1}(B)}^{q^{\prime} / p^{\prime}}\left\|w^{q^{\prime} / p}\right\|_{L_{\left(p^{\prime} / q^{\prime}\right)^{\prime}}(B)} .
$$

Moreover, we can write $w^{1-p^{\prime}} \in A_{p^{\prime} / q^{\prime}} \Rightarrow w^{1-p^{\prime}} \in A_{p^{\prime}}$ because of $w^{1-p^{\prime}} \in A_{p^{\prime} / q^{\prime}} \subset$ $A_{p^{\prime}}$. Therefore, we get

$$
\begin{aligned}
w^{1-p^{\prime}} \in A_{p^{\prime} / q^{\prime}} & \Rightarrow w^{1-p^{\prime}} \in A_{p^{\prime}} \\
& \Rightarrow\left[w^{1-p^{\prime}}\right]_{A_{p^{\prime}}(B)}^{1 / p^{\prime}}=|B|^{-1}\left\|w^{1-p^{\prime}}\right\|_{L_{1}(B)}^{1 / p^{\prime}}\left\|w^{1 / p}\right\|_{L_{p}(B)} .
\end{aligned}
$$

But the opposite is not true.

Remark 3.2 Let's write $w^{1-p^{\prime}} \in A_{p^{\prime} / q^{\prime}}$ and used the definitions $A_{p}$ classes we get the following

$$
\begin{aligned}
w^{1-p^{\prime}} \in A_{p^{\prime} / q^{\prime}} & \Rightarrow\left[w^{1-p^{\prime}}\right]_{A_{p^{\prime} / q^{\prime}}}^{\frac{q(p-1)}{p(q-1)}}=|B|^{-1}\left\|w^{1-p^{\prime}}\right\|_{L_{1}(B)}^{\frac{q(p-1)}{p(q-1)}}\left\|w^{q^{\prime} / p^{\prime}}\right\|_{L_{\left(p^{\prime} / q^{\prime}\right)^{\prime}}(B)} \\
& \Rightarrow\left[w^{1-p^{\prime}}\right]_{A_{p^{\prime} / q^{\prime}}^{1 / p^{\prime}}}=|B|^{-\frac{q-1}{q}}\left\|w^{1-p^{\prime}}\right\|_{L_{1}(B)}^{1 / p^{\prime}}\|w\|_{L_{\frac{q}{q}(B)}^{1 / p},}^{1-p},
\end{aligned}
$$

where the following equalities are provided.

$$
1-p^{\prime}=-\frac{p^{\prime}}{p}, \frac{q^{\prime}}{p}=\frac{q}{p(q-1)}, \frac{q^{\prime}}{p^{\prime}}=\frac{q(p-1)}{p(q-1)}, \quad\left(\frac{q}{p}\right)^{\prime}=\frac{q}{q-p}, \quad\left(\frac{p^{\prime}}{q^{\prime}}\right)^{\prime}=\frac{p(q-1)}{q-p} .
$$

Then from eq.(3.3) and eq.(3.4) we have

$$
\begin{aligned}
w^{1-p^{\prime}} \in A_{p^{\prime} / q^{\prime}} & \Rightarrow\left[w^{1-p^{\prime}}\right]_{A_{p^{\prime} / q^{\prime}}^{1 / p^{\prime}}}^{A^{\prime}} \\
& =|B|^{\frac{1}{q}}\left[w^{1-p^{\prime}}\right]_{A_{p^{\prime}}(B)}^{1 / p^{\prime}}\left\|w^{1 / p}\right\|_{L_{p}(B)}^{-1}\|w\|_{L_{\frac{q}{q-p}(B)}^{1 / p}}^{1} .
\end{aligned}
$$

We define the generalized weighed Morrey spaces as follows. 
Definition 3.1 [20] Let $1 \leq p<\infty$, $\varphi$ be a positive measurable function on $\mathbb{R}^{n} \times(0, \infty)$ and $w$ be non-negative measurable function on $\mathbb{R}^{n}$. We denote by $M_{p, \varphi}(w)$ the generalized weighted Morrey space, the space of all functions $f \in L_{p, w}^{\mathrm{loc}}\left(\mathbb{R}^{n}\right)$ with finite norm

$$
\|f\|_{M_{p, \varphi}(w)}=\sup _{x \in \mathbb{R}^{n}, r>0} \varphi(x, r)^{-1} w(B(x, r))^{-\frac{1}{p}}\|f\|_{L_{p, w}(B(x, r))},
$$

where $L_{p, w}(B(x, r))$ denotes the weighted $L_{p}$-space of measurable functions $f$ for which

$$
\|f\|_{L_{p, w}(B(x, r))} \equiv\left\|f \chi_{B(x, r)}\right\|_{L_{p, w}\left(\mathbb{R}^{n}\right)}=\left(\int_{B(x, r)}|f(y)|^{p} w(y) d y\right)^{\frac{1}{p}} .
$$

Furthermore, by $W M_{p, \varphi}(w)$ we denote the weak generalized weighted Morrey space of all functions $f \in W L_{p, w}^{\text {loc }}\left(\mathbb{R}^{n}\right)$ for which

$$
\|f\|_{W M_{p, \varphi}(w)}=\sup _{x \in \mathbb{R}^{n}, r>0} \varphi(x, r)^{-1} w(B(x, r))^{-\frac{1}{p}}\|f\|_{W L_{p, w}(B(x, r))}<\infty,
$$

where $W L_{p, w}(B(x, r))$ denotes the weak $L_{p, w}$-space of measurable functions $f$ for which

$$
\|f\|_{W L_{p, w}(B(x, r))} \equiv\left\|f \chi_{B(x, r)}\right\|_{W L_{p, w}\left(\mathbb{R}^{n}\right)}=\sup _{t>0} t\left(\int_{\{y \in B(x, r):|f(y)|>t\}} w(y) d y\right)^{\frac{1}{p}} .
$$

Remark 3.3 (1) If $w \equiv 1$, then $M_{p, \varphi}(1)=M_{p, \varphi}$ is the generalized Morrey space.

(2) If $\varphi(x, r) \equiv w(B(x, r))^{\frac{\kappa-1}{p}}$, then $M_{p, \varphi}(w)=L_{p, \kappa}(w)$ is the weighted Morrey space.

(3) If $\varphi(x, r) \equiv v(B(x, r))^{\frac{\kappa}{p}} w(B(x, r))^{-\frac{1}{p}}$, then $M_{p, \varphi}(w)=L_{p, \kappa}(v, w)$ is the two weighted Morrey space.

(4) If $w \equiv 1$ and $\varphi(x, r)=r^{\frac{\lambda-n}{p}}$ with $0<\lambda<n$, then $M_{p, \varphi}(w)=L_{p, \lambda}\left(\mathbb{R}^{n}\right)$ is the classical Morrey space and $W M_{p, \varphi}(w)=W L_{p, \lambda}\left(\mathbb{R}^{n}\right)$ is the weak Morrey space.

(5) If $\varphi(x, r) \equiv w(B(x, r))^{-\frac{1}{p}}$, then $M_{p, \varphi}(w)=L_{p, w}\left(\mathbb{R}^{n}\right)$ is the weighted Lebesgue space.

The following statement, was proved in [30].

Theorem 3.1 Let $1 \leq p<\infty, w \in A_{p}$ and $\left(\varphi_{1}, \varphi_{2}\right)$ satisfy the condition

$$
\sup _{t>r} \frac{\operatorname{ess~inf}_{t<\tau<\infty} \varphi_{1}(x, \tau) w(B(x, \tau))^{\frac{1}{p}}}{w(B(x, t))^{\frac{1}{p}}} \leq C \varphi_{2}(x, r),
$$

where $C$ does not depend on $x$ and $r$. Then the operator $M$ is bounded from $M_{p, \varphi_{1}}(w)$ to $M_{p, \varphi_{2}}(w)$ for $p>1$ and from $M_{1, \varphi_{1}}(w)$ to $W M_{1, \varphi_{2}}(w)$.

The following statement, was proved in [30], see also [20].

Theorem 3.2 Let $1<p<\infty, w \in A_{p}, b \in B M O\left(\mathbb{R}^{n}\right)$ and $\left(\varphi_{1}, \varphi_{2}\right)$ satisfy the condition

$$
\sup _{t>r}\left(1+\ln \frac{t}{r}\right) \frac{\underset{t<\tau<\infty}{\operatorname{essinf}} \varphi_{1}(x, \tau) w(B(x, \tau))^{\frac{1}{p}}}{w(B(x, t))^{\frac{1}{p}}} \leq C \varphi_{2}(x, r),
$$

where $C$ does not depend on $x$ and $r$. Then the operator $M_{b}$ is bounded from $M_{p, \varphi_{1}}(w)$ to $M_{p, \varphi_{2}}(w)$.

Note that, in the case $w=1$ Theorem 3.1 was proved in [23,37], see also [2]. 
4 Maximal operator with rough kernels $M_{\Omega}$ in the spaces $M_{p, \varphi}(w)$

In the following lemma we get Guliyev weighted local estimate (see, for example, [17, 19] in the case $w=1$ and [20] in the case $w \in A_{p}$ ) for the operator $T_{\Omega}$.

Lemma 4.1 Suppose that $\Omega$ be satisfies the condition (1.1) and $\Omega \in L_{q}\left(S^{n-1}\right), 1<q \leq$ $\infty$.

If $q^{\prime} \leq p<\infty, p \neq 1$ and $w \in A_{p / q^{\prime}}$, then the inequality

$$
\left\|M_{\Omega}(f)\right\|_{L_{p, w}(B(x, r))} \lesssim w(B(x, r))^{\frac{1}{p}} \sup _{t>2 r}\|f\|_{L_{p, w}(B(x, t))} w(B(x, t))^{-\frac{1}{p}}
$$

holds for any ball $B(x, r)$, and for all $f \in L_{p, w}^{\text {loc }}\left(\mathbb{R}^{n}\right)$.

If $1<p \leq q, p \neq \infty$ and $w^{1-p^{\prime}} \in A_{p^{\prime} / q^{\prime}}$, then the inequality

$$
\left\|M_{\Omega}(f)\right\|_{L_{p, w}(B(x, r))} \lesssim\|w\|_{L_{\frac{q}{q-p}(B(x, r))}^{1 / p}}^{1} \sup _{t>2 r}\|f\|_{L_{p, w}(B(x, t))}\|w\|_{L_{\frac{q}{q-p}(B(x, t))}^{-1 / p}}
$$

holds for any ball $B(x, r)$, and for all $f \in L_{p, w}^{\mathrm{loc}}\left(\mathbb{R}^{n}\right)$.

Proof. Let $\Omega$ be satisfies the condition (1.1) and $\Omega \in L_{q}\left(S^{n-1}\right), 1<q \leq \infty$.

Note that

$$
\|\Omega(x-\cdot)\|_{L_{q}(B(x, t))} \leq c_{0}\|\Omega\|_{L_{q}\left(S^{n-1}\right)}\left|B\left(0, t+\left|x-x_{0}\right|\right)\right|^{\frac{1}{q}},
$$

where $c_{0}=\left(n v_{n}\right)^{-1 / q}$ and $v_{n}=|B(0,1)|$ (see, [23]).

For arbitrary $x_{0} \in \mathbb{R}^{n}$, set $B=B(x, r)$ for the ball centered at $x_{0}$ and of radius $r$, $2 B=B\left(x_{0}, 2 r\right)$. We represent $f$ as

$$
f=f_{1}+f_{2}, \quad f_{1}(y)=f(y) \chi_{2 B}(y), \quad f_{2}(y)=f(y) \chi_{\mathrm{c}_{(2 B)}}(y), \quad r>0
$$

and have

$$
\left\|M_{\Omega}(f)\right\|_{L_{p, w}(B)} \leq\left\|M_{\Omega}\left(f_{1}\right)\right\|_{L_{p, w}(B)}+\left\|M_{\Omega}\left(f_{2}\right)\right\|_{L_{p, w}(B)} .
$$

Since $f_{1} \in L_{p, w}\left(\mathbb{R}^{n}\right), M_{\Omega}\left(f_{1}\right) \in L_{p, w}\left(\mathbb{R}^{n}\right)$ and from the boundedness of $M_{\Omega}$ in $L_{p, w}\left(\mathbb{R}^{n}\right)$ for $w \in A_{p / q^{\prime}}$ and $q^{\prime} \leq p<\infty, p \neq 1$ (see Theorem 2.2) it follows that

$$
\begin{aligned}
\left\|M_{\Omega}\left(f_{1}\right)\right\|_{L_{p, w}(B)} & \leq\left\|M_{\Omega}\left(f_{1}\right)\right\|_{L_{p, w}\left(\mathbb{R}^{n}\right)} \\
& \lesssim\|\Omega\|_{L_{q}\left(S^{n-1}\right)}[w]_{A_{\frac{p}{q^{\prime}}}^{\frac{1}{p}}}^{p}\left\|f_{1}\right\|_{L_{p, w}\left(\mathbb{R}^{n}\right)} \\
& \approx\|\Omega\|_{L_{q}\left(S^{n-1}\right)}[w]_{A_{\frac{p}{q^{\prime}}}^{\frac{1}{p}}}\|f\|_{L_{p, w}(2 B)} \\
& \lesssim\|\Omega\|_{L_{q}\left(S^{n-1}\right)}[w]_{A_{\frac{p}{q^{\prime}}}^{\frac{1}{p}}} w(B)^{\frac{1}{p}} \sup _{t>2 r}\|f\|_{L_{p, w}(B(x, t))} w(B(x, t))^{-\frac{1}{p}}
\end{aligned}
$$

Let $z$ be an arbitrary point in $B \equiv B(x, r)$. If $B(z, t) \cap{ }^{\complement} B(x, 2 r) \neq \varnothing$, then $t>r$. Indeed, if $y \in B(z, t) \cap{ }^{C} B(x, 2 r)$, then we get $t>|y-z| \geq|x-y|-|x-z|>2 r-r=r$. 
On the other hand, $B(z, t) \cap{ }^{\mathrm{c}} B(x, 2 r) \subset B(x, 2 t)$. Indeed, if $y \in B(z, t) \cap{ }^{\mathrm{C}} B(x, 2 r)$, then we get $|x-y| \leq|y-z|+|x-z|<t+r<2 t$. Hence, for all $z \in B$

$$
\begin{aligned}
M_{\Omega} f_{2}(z) & =\sup _{t>0}|B(z, t)|^{-1} \int_{B(z, t)}|\Omega(z-y)|\left|f_{2}(y)\right| d y \\
& \leq \sup _{t>r}|B(x, 2 t)|^{-1} \int_{B(z, t) \cap^{\complement} B(x, 2 r)}|\Omega(z-y)||f(y)| d y \\
& \leq \sup _{t>r}|B(x, 2 t)|^{-1} \int_{B(x, 2 t)}|\Omega(z-y)||f(y)| d y \\
& =\sup _{t>2 r}|B(x, t)|^{-1} \int_{B(x, t)}|\Omega(z-y)||f(y)| d y .
\end{aligned}
$$

By applying Hölder's inequality for $q^{\prime} \leq p<\infty, p \neq 1$ and $w \in A_{p / q^{\prime}}$, we get

$$
\begin{aligned}
& M_{\Omega} f_{2}(z) \leq \sup _{t>2 r}|B(x, t)|^{-1} \int_{B(x, t)}|\Omega(z-y)||f(y)| d y \\
& \lesssim \sup _{t>2 r}|B(x, t)|^{-1}\|\Omega(z-\cdot)\|_{L_{q}(B(x, t))}\|f\|_{L_{q^{\prime}}(B(x, t))} \\
& \lesssim\|\Omega\|_{L_{q}\left(S^{n-1}\right)} \sup _{t>2 r}|B(x, t)|^{-1}\|f\|_{L_{p, w}(B(x, t))}\left\|w^{-q^{\prime} / p}\right\|_{L_{\left(p / q^{\prime}\right)^{\prime}}(B(x, t))}^{\frac{1}{q^{\prime}}}|B(0, t+|x-z|)|^{\frac{1}{q}} \\
& \lesssim\|\Omega\|_{L_{q}\left(S^{n-1}\right)}[w]_{A_{q^{\prime}}}^{\frac{1}{p}} \sup _{t>2 r}|B(x, t)|^{-1}\|f\|_{L_{p, w}(B(x, t))} w(B(x, t))^{-\frac{1}{p}}|B(x, t)|^{\frac{1}{q^{\prime}}}|B(0, t+r)|^{\frac{1}{q}} \\
& \approx\|\Omega\|_{L_{q}\left(S^{n-1}\right)}[w]_{A_{q^{\prime}}}^{\frac{p}{p}} \sup _{t>2 r}\|f\|_{L_{p, w}(B(x, t))} w(B(x, t))^{-\frac{1}{p}} .
\end{aligned}
$$

Moreover, for all $q^{\prime} \leq p<\infty, p \neq 1$ the inequality

$$
\left\|M_{\Omega}\left(f_{2}\right)\right\|_{L_{p, w}(B)} \lesssim\|\Omega\|_{L_{q}\left(S^{n-1}\right)}[w]_{A_{q^{\prime}}}^{\frac{1}{p}} w(B)^{\frac{1}{p}} \sup _{t>2 r}\|f\|_{L_{p, w}(B(x, t))} w(B(x, t))^{-\frac{1}{p}}
$$

is valid. Thus

$$
\left\|M_{\Omega}(f)\right\|_{L_{p, w}(B)} \lesssim\|\Omega\|_{L_{q}\left(S^{n-1}\right)}[w]_{A_{q^{\prime}}}^{\frac{1}{p}} w(B)^{\frac{1}{p}} \sup _{t>2 r}\|f\|_{L_{p, w}(B(x, t))} w(B(x, t))^{-\frac{1}{p}}
$$

Let also $1<p \leq q, p \neq \infty$ and $w^{1-p^{\prime}} \in A_{p^{\prime} / q^{\prime}}$. Since $f_{1} \in L_{p, w}\left(\mathbb{R}^{n}\right), M_{\Omega}\left(f_{1}\right) \in$ $L_{p, w}\left(\mathbb{R}^{n}\right)$ and from the boundedness of $M_{\Omega}$ in $L_{p, w}\left(\mathbb{R}^{n}\right)$ for $w^{1-p^{\prime}} \in A_{p^{\prime}} / q^{\prime}$ and $1<p<q$ (see Theorem 2.2) it follows that

$$
\begin{aligned}
\left\|M_{\Omega}\left(f_{1}\right)\right\|_{L_{p, w}(B)} & \leq\left\|M_{\Omega}\left(f_{1}\right)\right\|_{L_{p, w}\left(\mathbb{R}^{n}\right)} \lesssim\|\Omega\|_{L_{q}\left(S^{n-1}\right)}\left[w^{1-p^{\prime}}\right]_{A_{\frac{p^{\prime}}{q^{\prime}}}^{\frac{1}{p^{\prime}}}}\left\|f_{1}\right\|_{L_{p, w}\left(\mathbb{R}^{n}\right)} \\
& \approx\|\Omega\|_{L_{q}\left(S^{n-1}\right)}\left[w^{1-p^{\prime}}\right]_{\frac{p_{q^{\prime}}}{p^{\prime}}}^{\frac{1}{p^{\prime}}}\|f\|_{L_{p, w}(2 B)} .
\end{aligned}
$$


If $1<p \leq q, p \neq \infty$ and $w^{1-p^{\prime}} \in A_{p^{\prime} / q^{\prime}}$, then Minkowski theorem and Hölder inequality,

$$
\begin{aligned}
& \left\|M_{\Omega}\left(f_{2}\right)\right\|_{L_{p, w}(B)} \leq\left(\int_{B}\left(\sup _{t>2 r}|B(x, t)|^{-1} \int_{B(x, t)}|\Omega(x-y)||f(y)| d y\right)^{p} w(x) d x\right)^{\frac{1}{p}} \\
& \leq \sup _{t>2 r}|B(x, t)|^{-1} \int_{B(x, t)}\|\Omega(\cdot-y)\|_{L_{p, w}(B)}|f(y)| d y \\
& \lesssim \sup _{t>2 r}|B(x, t)|^{-1} \int_{B(x, t)}\|\Omega(\cdot-y)\|_{L_{q}(B)}\|w\|_{L_{(q / p)^{\prime}}(B)}^{\frac{1}{p}}|f(y)| d y \\
& \lesssim\|\Omega\|_{L_{q}\left(S^{n-1}\right)}\|w\|_{L_{(q / p)^{\prime}}(B)}^{\frac{1}{p}} \sup _{t>2 r}|B(x, t)|^{-1} \int_{B(x, t)}|B(0, r+|x-y|)|^{\frac{1}{q}}|f(y)| d y \\
& \lesssim\|\Omega\|_{L_{q}\left(S^{n-1}\right)}\|w\|_{L_{(q / p)^{\prime}}(B)}^{\frac{1}{p}} \sup _{t>2 r}|B(x, t)|^{-1}\|f\|_{L_{1}(B(x, t))}|B(0, r+t)|^{\frac{1}{q}} \\
& \lesssim\|\Omega\|_{L_{q}\left(S^{n-1}\right)}\|w\|_{L_{\frac{q}{q-p}}^{\frac{1}{p}}(B)} \sup _{t>2 r}|B(x, t)|^{-1}\|f\|_{L_{p, w}(B(x, t))}\left\|w^{-p^{\prime} / p}\right\|_{L_{1}(B(x, t))}^{\frac{1}{p^{\prime}}}|B(x, t)|^{\frac{1}{q}} \\
& \lesssim\|\Omega\|_{L_{q}\left(S^{n-1}\right)}\|w\|_{L_{\frac{q}{q-p}}^{\frac{1}{p}}(B)} \sup _{t>2 r}|B(x, t)|^{-1}\|f\|_{L_{p, w}(B(x, t))}\left\|w^{1-p^{\prime}}\right\|_{L_{1}(B(x, t))}^{\frac{1}{p^{\prime}}}|B(x, t)|^{\frac{1}{q}}
\end{aligned}
$$

is obtained. By applying (3.3) for $\left\|w^{1-p^{\prime}}\right\|_{L_{1}(B(x, t))}^{\frac{1}{p^{\prime}}}$ and (3.5) for $\|w\|_{L_{\frac{q}{q-p}}^{\frac{1}{p}}}^{\frac{1}{p}(B)}$ we have the following inequality

$$
\begin{aligned}
& \left\|M_{\Omega}\left(f_{2}\right)\right\|_{L_{p, w}(B)} \\
& \lesssim\|\Omega\|_{L_{q}\left(S^{n-1}\right)}\left[w^{1-p^{\prime}}\right]_{A_{\frac{p^{\prime}}{q^{\prime}}}^{\frac{1}{p^{\prime}}}}^{\frac{1}{p^{\prime}}}\|w\|_{L_{\frac{q}{q-p}(B)}^{\frac{1}{p}}} \sup _{t>2 r}\|f\|_{L_{p, w}(B(x, t))}\|w\|_{L_{\frac{q}{q-p}}^{-\frac{1}{p}}(B(x, t))}
\end{aligned}
$$

is valid. Thus

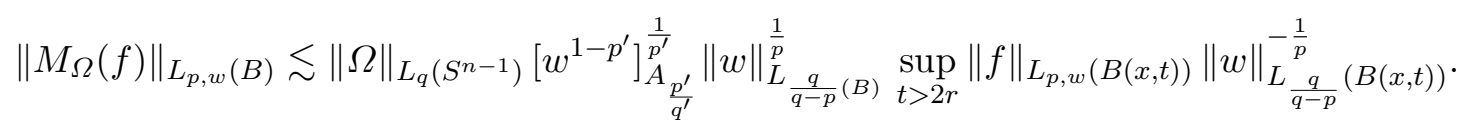

Thus we complete the proof of Lemma 4.1.

Theorem 4.1 Suppose that $\Omega$ be satisfies the condition (1.1) and $\Omega \in L_{q}\left(S^{n-1}\right), 1<q \leq$ $\infty$. Let also, for $q^{\prime} \leq p<\infty, w \in A_{p / q^{\prime}}$ the pair $\left(\varphi_{1}, \varphi_{2}\right)$ satisfies the condition (3.6) and for $1<p \leq q, w^{1-p^{\prime}} \in A_{p^{\prime} / q^{\prime}}$ the pair $\left(\varphi_{1}, \varphi_{2}\right)$ satisfies the condition

$$
\sup _{t>r} \frac{\underset{t<\tau<\infty}{\operatorname{ess} \inf _{t<}} \varphi_{1}(x, \tau)\|w\|_{L_{\frac{q}{q-p}(B(x, \tau))}^{1 / p}}^{1 / p}}{\|w\|_{L_{\frac{q}{q-p}(B(x, t))}}^{1 / p}} \leq C \varphi_{2}(x, r) \frac{w(B(x, r))^{\frac{1}{p}}}{\|w\|_{L_{\frac{q}{q-p}(B(x, r))}^{\frac{1}{p}}}^{p}},
$$

where $C$ does not depend on $x$ and $r$.

Then the operator $M_{\Omega}$ is bounded from $M_{p, \varphi_{1}}(w)$ to $M_{p, \varphi_{2}}(w)$. Moreover

$$
\left\|M_{\Omega}(f)\right\|_{M_{p, \varphi_{2}}(w)} \lesssim\|f\|_{M_{p, \varphi_{1}}(w)} .
$$


Proof. When $q^{\prime} \leq p<\infty, w \in A_{p / q^{\prime}}$, by Lemma 4.1 and Theorem 2.3 with $\nu_{2}(r)=$ $\varphi_{2}(x, r)^{-1}, \nu_{1}(r)=\varphi_{1}(x, r)^{-1} w(B(x, r))^{-\frac{1}{p}}, g(r)=\|f\|_{L_{p, w}(B(x, r))}$ and $w(r)=w(B(x, r))^{-\frac{1}{p}}$ we have

$$
\begin{aligned}
\left\|M_{\Omega}(f)\right\|_{M_{p, \varphi_{2}}(w)} & =\sup _{x \in \mathbb{R}^{n}, r>0} \varphi_{2}(x, r)^{-1} w(B(x, r))^{-\frac{1}{p}}\left\|M_{\Omega}(f)\right\|_{L_{p, w}(B(x, r))} \\
& \lesssim \sup _{x \in \mathbb{R}^{n}, r>0} \varphi_{2}(x, r)^{-1} \sup _{t>r}\|f\|_{L_{p, w}(B(x, t))} w(B(x, t))^{-\frac{1}{p}} \\
& \lesssim \sup _{x \in \mathbb{R}^{n}, r>0} \varphi_{1}(x, r)^{-1} w(B(x, r))^{-\frac{1}{p}}\|f\|_{L_{p, w}(B(x, r))} \\
& =\|f\|_{M_{p, \varphi_{1}}(w) .}
\end{aligned}
$$

For the case of $1<p \leq q, w^{1-p^{\prime}} \in A_{p^{\prime} / q^{\prime}}$, by Lemma 4.1 and Theorem 2.3 with

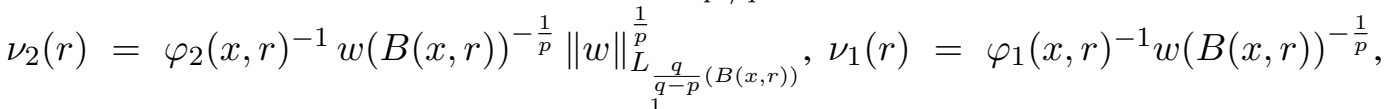

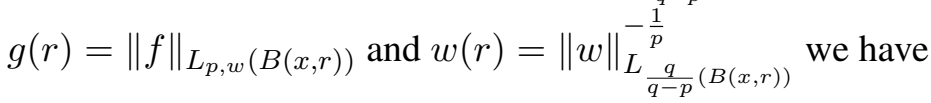

$$
\begin{aligned}
& \left\|M_{\Omega}(f)\right\|_{M_{p, \varphi_{2}}(w)}=\sup _{x \in \mathbb{R}^{n}, r>0} \varphi_{2}(x, r)^{-1} w(B(x, r))^{-\frac{1}{p}}\left\|M_{\Omega}(f)\right\|_{L_{p, w}(B(x, r))} \\
& \lesssim \sup _{x \in \mathbb{R}^{n}, r>0} \varphi_{2}(x, r)^{-1} w(B(x, r))^{-\frac{1}{p}}\|w\|_{L_{\frac{q}{q-p}(B)}^{\frac{1}{p}}} \sup _{t>r}\|f\|_{L_{p, w}(B(x, t))}\|w\|_{L_{\frac{q}{q-p}(B(x, t))}^{-\frac{1}{p}}} \\
& \lesssim \sup _{x \in \mathbb{R}^{n}, r>0} \varphi_{1}(x, r)^{-1} w(B(x, r))^{-\frac{1}{p}}\|f\|_{L_{p, w}(B(x, r))} \\
& =\|f\|_{M_{p, \varphi_{1}}(w) .}
\end{aligned}
$$

5 Commutator of maximal operator with rough kernels $\left[b, M_{\Omega}\right]$ in the spaces $M_{p, \varphi}(w)$

We recall the definition of the space of $B M O\left(\mathbb{R}^{n}\right)$.

Definition 5.1 Suppose that $b \in L_{1}^{\text {loc }}\left(\mathbb{R}^{n}\right)$, and let

$$
\|b\|_{*}=\sup _{x \in \mathbb{R}^{n}, r>0} \frac{1}{|B(x, r)|} \int_{B(x, r)}\left|b(y)-b_{B(x, r)}\right| d y<\infty,
$$

where

$$
b_{B(x, r)}=\frac{1}{|B(x, r)|} \int_{B(x, r)} b(y) d y .
$$

Define

$$
B M O\left(\mathbb{R}^{n}\right)=\left\{b \in L_{1}^{\text {loc }}\left(\mathbb{R}^{n}\right):\|b\|_{*}<\infty\right\} .
$$

Modulo constants, the space $B M O\left(\mathbb{R}^{n}\right)$ is a Banach space with respect to the norm $\|\cdot\|_{*}$.

Lemma 5.1 [36] Let $w \in A_{\infty}$. Then the norm $\|\cdot\|_{*}$ is equivalent to the norm

$$
\|b\|_{*, w}=\sup _{x \in \mathbb{R}^{n}, r>0} \frac{1}{w(B(x, r))} \int_{B(x, r)}\left|b(y)-b_{B(x, r), w}\right| w(y) d y,
$$

where

$$
b_{B(x, r), w}=\frac{1}{w(B(x, r))} \int_{B(x, r)} b(y) w(y) d y .
$$


The following lemma is proved in [20].

Lemma 5.2 1 Let $w \in A_{\infty}$ and $b \in B M O\left(\mathbb{R}^{n}\right)$. Let also $1 \leq p<\infty, x \in \mathbb{R}^{n}$ and $r_{1}, r_{2}>0$. Then,

$$
\left(\frac{1}{w\left(B\left(x, r_{1}\right)\right)} \int_{B\left(x, r_{1}\right)}\left|b(y)-b_{B\left(x, r_{2}\right), w}\right|^{p} w(y) d y\right)^{\frac{1}{p}} \leq C\left(1+\left|\ln \frac{r_{1}}{r_{2}}\right|\right)\|b\|_{*},
$$

where $C>0$ is independent of $f, w, x, r_{1}$ and $r_{2}$.

2 Let $w \in A_{p}$ and $b \in B M O\left(\mathbb{R}^{n}\right)$. Let also $1<p<\infty, x \in \mathbb{R}^{n}$ and $r_{1}, r_{2}>0$. Then,

$$
\begin{aligned}
\left(\frac{1}{w^{1-p^{\prime}}\left(B\left(x, r_{1}\right)\right)} \int_{B\left(x, r_{1}\right)}\left|b(y)-b_{B\left(x, r_{2}\right), w}\right|^{p^{\prime}}\right. & \left.w(y)^{1-p^{\prime}} d y\right)^{\frac{1}{p^{\prime}}} \\
& \leq C\left(1+\left|\ln \frac{r_{1}}{r_{2}}\right|\right)\|b\|_{*},
\end{aligned}
$$

where $C>0$ is independent of $b, w, x, r_{1}$ and $r_{2}$.

\section{Remark 5.1 ([27])}

(1) The John-Nirenberg inequality : There are constants $C_{1}, C_{2}>0$, such that for all $b \in B M O\left(\mathbb{R}^{n}\right)$ and $\beta>0$

$$
\left|\left\{x \in B:\left|b(x)-b_{B}\right|>\beta\right\}\right| \leq C_{1}|B| e^{-C_{2} \beta /\|b\|_{*}}, \quad \forall B \subset \mathbb{R}^{n} .
$$

(2) The John-Nirenberg inequality implies that

$$
\|b\|_{*} \approx \sup _{x \in \mathbb{R}^{n}, r>0}\left(\frac{1}{|B(x, r)|} \int_{B(x, r)}\left|b(y)-b_{B(x, r)}\right|^{p} d y\right)^{\frac{1}{p}}
$$

for $1<p<\infty$.

(3) Let $b \in B M O\left(\mathbb{R}^{n}\right)$. Then there is a constant $C>0$ such that

$$
\left|b_{B(x, r)}-b_{B(x, t)}\right| \leq C\|b\|_{*} \ln \frac{t}{r} \text { for } 0<2 r<t,
$$

where $C$ is independent of $b, x, r$ and $t$.

In the following lemma we get Guliyev weighted local estimate (see, for example, [20] ) for the maximal commutator operator $M_{\Omega, b}$.

Lemma 5.3 Let $1<p<\infty$ and $b \in B M O\left(\mathbb{R}^{n}\right)$. Suppose that $\Omega$ be satisfies the condition (1.1) and $\Omega \in L_{q}\left(S^{n-1}\right), 1<q \leq \infty$.

If $q^{\prime} \leq p<\infty$ and $w \in A_{p / q^{\prime}}$, then the inequality

$$
\begin{aligned}
& \left\|M_{\Omega, b}(f)\right\|_{L_{p, w}(B(x, r))} \\
& \lesssim\|b\|_{*} w(B(x, r))^{\frac{1}{p}} \sup _{t>2 r}\left(1+\ln \frac{t}{r}\right)\|f\|_{L_{p, w}(B(x, t))} w(B(x, t))^{-\frac{1}{p}}
\end{aligned}
$$

holds for any ball $B(x, r)$, and for all $f \in L_{p, w}^{\text {loc }}\left(\mathbb{R}^{n}\right)$.

If $1<p<q$ and $w^{1-p^{\prime}} \in A_{p^{\prime} / q^{\prime}}$, then the inequality

$$
\begin{aligned}
& \left\|M_{\Omega, b}(f)\right\|_{L_{p, w}(B(x, r))} \\
& \lesssim\|w\|_{L_{\frac{q}{q-p}(B(x, r))}}^{\frac{1}{p}} \sup _{t>2 r}\left(1+\ln \frac{t}{r}\right)\|f\|_{L_{p, w}(B(x, t))}\|w\|_{L_{\frac{q}{q-p}(B(x, t))}^{-\frac{1}{p}}}
\end{aligned}
$$

holds for any ball $B(x, r)$, and for all $f \in L_{p, w}^{\text {loc }}\left(\mathbb{R}^{n}\right)$. 
Proof. Let $p \in(1, \infty)$ and $b \in B M O\left(\mathbb{R}^{n}\right)$. For arbitrary $x_{0} \in \mathbb{R}^{n}$, set $B=B(x, r)$ for the ball centered at $x$ and of radius $r, 2 B=B(x, 2 r)$. We represent $f$ as (4.2) and have

$$
\left\|M_{\Omega, b}(f)\right\|_{L_{p, w}(B)} \leq\left\|M_{\Omega, b}\left(f_{1}\right)\right\|_{L_{p, w}(B)}+\left\|M_{\Omega, b}\left(f_{2}\right)\right\|_{L_{p, w}(B)} .
$$

Since $f_{1} \in L_{p, w}\left(\mathbb{R}^{n}\right), M_{\Omega, b}\left(f_{1}\right) \in L_{p, w}\left(\mathbb{R}^{n}\right)$ and from the boundedness of $M_{\Omega, b}$ in $L_{p, w}\left(\mathbb{R}^{n}\right)$ for $w \in A_{p / q^{\prime}}$ and $q^{\prime} \leq p<\infty$ (see Theorem 2.2) it follows that

$$
\begin{aligned}
\left\|M_{\Omega, b}\left(f_{1}\right)\right\|_{L_{p, w}(B)} & \leq\left\|M_{\Omega, b}\left(f_{1}\right)\right\|_{L_{p, w}\left(\mathbb{R}^{n}\right)} \\
& \lesssim\|\Omega\|_{L_{q}\left(S^{n-1}\right)}[w]_{A_{\frac{p}{q^{\prime}}}^{p}}^{\frac{1}{p}}\|b\|_{*}\left\|f_{1}\right\|_{L_{p, w}\left(\mathbb{R}^{n}\right)} \\
& \approx\|\Omega\|_{L_{q}\left(S^{n-1}\right)}[w]_{A_{\frac{p}{q^{\prime}}}^{p}}^{\frac{1}{p}}\|b\|_{*}\|f\|_{L_{p, w}(2 B)} .
\end{aligned}
$$

Let $z$ be an arbitrary point in $B \equiv B(x, r)$. If $B(z, t) \cap{ }^{\mathrm{C}} B(x, 2 r) \neq \varnothing$, then $t>r$. Indeed, if $y \in B(z, t) \cap{ }^{\mathrm{C}} B(x, 2 r)$, then we get $t>|y-z| \geq|x-y|-|x-z|>2 r-r=r$.

On the other hand, $B(z, t) \cap{ }^{\mathrm{C}} B(x, 2 r) \subset B(x, 2 t)$. Indeed, if $y \in B(z, t) \cap{ }^{\mathrm{C}} B(x, 2 r)$, then we get $|x-y| \leq|y-z|+|x-z|<t+r<2 t$. Hence, for all $z \in B$

$$
\begin{aligned}
M_{\Omega, b} f_{2}(z) & =\sup _{t>0}|B(z, t)|^{-1} \int_{B(z, t)}|b(y)-b(z)||\Omega(y-z)|\left|f_{2}(y)\right| d y \\
& =\sup _{t>0}|B(z, t)|^{-1} \int_{B(z, t) \cap^{\complement} B(x, 2 r)}|b(y)-b(z)||\Omega(y-z)||f(y)| d y \\
& \lesssim \sup _{t>r}|B(x, 2 t)|^{-1} \int_{B(x, 2 t)}|b(y)-b(z)||\Omega(y-z)||f(y)| d y \\
& =\sup _{t>2 r}|B(x, 2 t)|^{-1} \int_{B(x, t)}|b(y)-b(z)||\Omega(y-z)||f(y)| d y .
\end{aligned}
$$

Therefore, for all $z \in B$ we have

$$
M_{\Omega, b} f_{2}(z) \lesssim \sup _{t>2 r}|B(x, 2 t)|^{-1} \int_{B(x, t)}|b(y)-b(z)||\Omega(y-z)||f(y)| d y .
$$

By applying Hölder's inequality for $q^{\prime} \leq p<\infty, p \neq 1$ and $w \in A_{p / q^{\prime}}$, we get

$$
\begin{aligned}
& M_{\Omega, b} f_{2}(z) \leq \sup _{t>2 r}|B(x, t)|^{-1} \int_{B(x, t)}|b(y)-b(z)||\Omega(z-y)||f(y)| d y \\
& \lesssim \sup _{t>2 r}|B(x, t)|^{-1}\|\Omega(z-\cdot)\|_{L_{q}(B(x, t))}\|(b(y)-b(z)) f\|_{L_{q^{\prime}}(B(x, t))} \\
& \left.\lesssim\|\Omega\|_{L_{q}\left(S^{n-1}\right)} \sup _{t>2 r}|B(x, t)|^{-1}\|f\|_{L_{p, w}(B(x, t))}\left\|(b(y)-b(z)) w^{-q^{\prime} / p}\right\|_{L_{\left(p / q^{\prime}\right)^{\prime}}}^{\frac{1}{q^{\prime}}} \mid B(x, t)\right) \\
& \lesssim\|\Omega\|_{L_{q}\left(S^{n-1}\right)}[w]_{A \frac{p}{q^{\prime}}}^{\frac{1}{p}} \sup _{t>2 r}|B(x, t)|^{-1}\|f\|_{L_{p, w}(B(x, t))} w(B(x, t))^{-\frac{1}{p}}|B(x, t)|^{\frac{1}{q^{\prime}}}|B(0, t+r)|^{\frac{1}{q}} \\
& \approx\|\Omega\|_{L_{q}\left(S^{n-1}\right)}[w]_{A \frac{p}{q^{\prime}}}^{\frac{1}{p}} \sup _{t>2 r}\|f\|_{L_{p, w}(B(x, t))} w(B(x, t))^{-\frac{1}{p}}
\end{aligned}
$$

Moreover, for all $q^{\prime} \leq p<\infty, p \neq 1$ the inequality

$$
\left\|M_{\Omega}\left(f_{2}\right)\right\|_{L_{p, w}(B)} \lesssim\|\Omega\|_{L_{q}\left(S^{n-1}\right)}[w]_{A_{q^{\prime}}}^{\frac{1}{p}} w(B)^{\frac{1}{p}} \sup _{t>2 r}\|f\|_{L_{p, w}(B(x, t))} w(B(x, t))^{-\frac{1}{p}}
$$


is valid. Thus

$$
\left\|M_{\Omega}(f)\right\|_{L_{p, w}(B)} \lesssim\|\Omega\|_{L_{q}\left(S^{n-1}\right)}[w]_{A_{q^{\prime}}}^{\frac{1}{p}} w(B)^{\frac{1}{p}} \sup _{t>2 r}\|f\|_{L_{p, w}(B(x, t))} w(B(x, t))^{-\frac{1}{p}} .
$$

If $1<p \leq q, p \neq \infty$ and $w^{1-p^{\prime}} \in A_{p^{\prime} / q^{\prime}}$, then Minkowski theorem and Hölder inequality,

$$
\begin{aligned}
& \left\|M_{\Omega, b} f_{2}\right\|_{L_{p, w}(B)} \\
& \lesssim\left(\int_{B}\left(\sup _{t>2 r}|B(x, t)|^{-1} \int_{B(x, t)}|b(y)-b(z)||\Omega(y-z)||f(y)| d y\right)^{p} w(z) d z\right)^{\frac{1}{p}} \\
& \lesssim\left(\int_{B}\left(\sup _{t>2 r}|B(x, t)|^{-1} \int_{B(x, t)}\left|b(y)-b_{B, w}\right||\Omega(y-z)||f(y)| d y\right)^{p} w(z) d z\right)^{\frac{1}{p}} \\
& +\left(\int_{B}\left(\sup _{t>2 r}|B(x, t)|^{-1} \int_{B(x, t)}\left|b(z)-b_{B, w}\right||\Omega(y-z)||f(y)| d y\right)^{p} w(z) d z\right)^{\frac{1}{p}} \\
& =J_{1}+J_{2} .
\end{aligned}
$$

Let us estimate $J_{1}$. Applying Hölder's inequality and by Lemma 5.2 we get

$$
\begin{aligned}
& J_{1}=\left(\int_{B}\left(\sup _{t>2 r}|B(x, t)|^{-1} \int_{B(x, t)}\left|b(y)-b_{B, w}\right||\Omega(y-z)||f(y)| d y\right)^{p} w(z) d z\right)^{\frac{1}{p}} \\
& \leq \sup _{t>2 r}|B(x, t)|^{-1} \int_{B(x, t)}\|\Omega(y-\cdot)\|_{L_{p, w}(B)}\left|b(y)-b_{B, w}\right||f(y)| d y \\
& \lesssim \sup _{t>2 r}|B(x, t)|^{-1} \int_{B(x, t)}\|\Omega(y-\cdot)\|_{L_{q}(B)}\|w\|_{L_{(q / p)^{\prime}}}^{\frac{1}{p}}\left|b(y)-b_{B, w}\right||f(y)| d y \\
& \lesssim\|\Omega\|_{L_{q}\left(S^{n-1}\right)}\|w\|_{L_{(q / p)^{\prime}}(B)}^{\frac{1}{p}} \sup _{t>2 r}|B(x, t)|^{-1} \int_{B(x, t)}|B(0, r+|x-y|)|^{\frac{1}{q}}\left|b(y)-b_{B, w}\right||f(y)| d y \\
& \lesssim\|\Omega\|_{L_{q}\left(S^{n-1}\right)}\|w\|_{L_{(q / p)^{\prime}}(B)}^{\frac{1}{p}} \sup _{t>2 r}|B(x, t)|^{-1+\frac{1}{q}} \int_{B(x, t)}\left|b(y)-b_{B, w}\right||f(y)| d y \\
& \leq\|\Omega\|_{L_{q}\left(S^{n-1}\right)}\|w\|_{L_{(q / p)^{\prime}}(B)}^{\frac{1}{p}} \sup _{t>2 r}|B(x, t)|^{-1+\frac{1}{q}}\left(\int_{B(x, t)}\left|b(y)-b_{B, w}\right|^{p^{\prime}} w(y)^{1-p^{\prime}} d y\right)^{\frac{1}{p^{\prime}}}\|f\|_{L_{p, w}(B(x, t))} \\
& \lesssim\|b\|_{*}\|\Omega\|_{L_{q}\left(S^{n-1}\right)}\|w\|_{L_{(q / p)^{\prime}}(B)}^{\frac{1}{p}} \sup _{t>2 r}|B(x, t)|^{-1+\frac{1}{q}}\left(1+\ln \frac{t}{r}\right)\left\|w^{1-p^{\prime}}\right\|_{L_{1}(B(x, t))}^{\frac{1}{p^{\prime}}}\|f\|_{L_{p, w}(B(x, t))}
\end{aligned}
$$

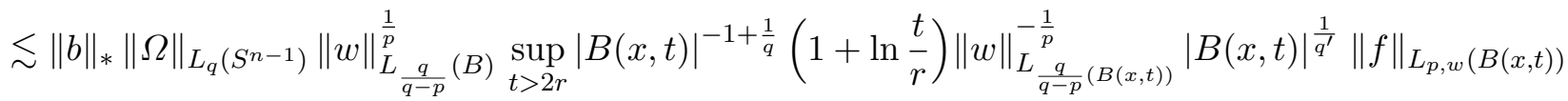

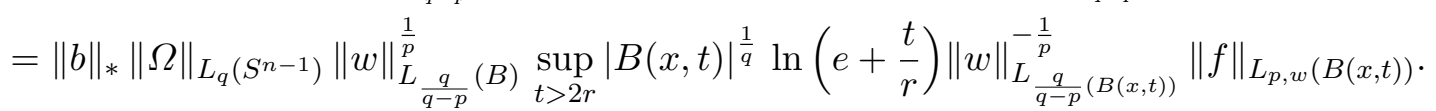

In order to estimate $J_{2}$ note that

$$
\begin{aligned}
J_{2} & =\left(\int_{B}\left(\sup _{t>2 r}|B(x, t)|^{-1} \int_{B(x, t)}\left|b(z)-b_{B, w}\right||\Omega(y-z)||f(y)| d y\right)^{p} w(z) d z\right)^{\frac{1}{p}} \\
& \leq \sup _{t>2 r}|B(x, t)|^{-1} \int_{B(x, t)}\left(\int_{B}\left|\left(b(z)-b_{B, w}\right) \Omega(y-z)\right|^{p} w(z) d z\right)^{\frac{1}{p}}|f(y)| d y .
\end{aligned}
$$

With similar techniques for $1<p \leq q, w^{1-p^{\prime}} \in A_{p^{\prime}} / q^{\prime}$ can be achieved and the proof is finished. 
Theorem 5.1 Suppose that $\Omega$ be satisfies the condition (1.1) and $\Omega \in L_{q}\left(S^{n-1}\right), 1<q \leq$ $\infty$. Let $b \in B M O\left(\mathbb{R}^{n}\right)$. Let also, for $q^{\prime} \leq p<\infty, w \in A_{p / q^{\prime}}$ the pair $\left(\varphi_{1}, \varphi_{2}\right)$ satisfies the condition (3.7) and for $1<p \leq q, w^{1-p^{\prime}} \in A_{p^{\prime}} / q^{\prime}$ the pair $\left(\varphi_{1}, \varphi_{2}\right)$ satisfies the condition

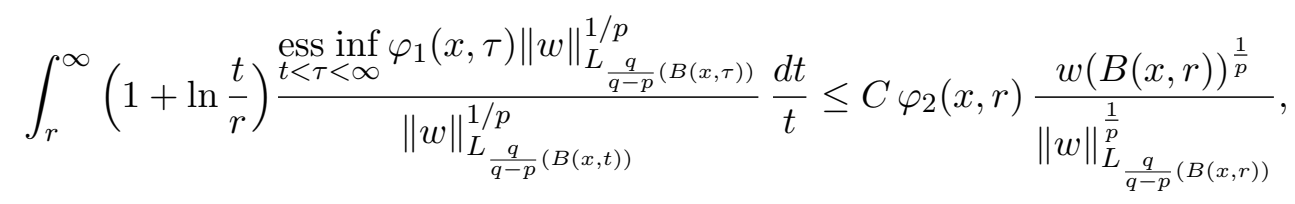

where $C$ does not depend on $x$ and $r$.

Then the operator $M_{\Omega, b}$ is bounded from $M_{p, \varphi_{1}}(w)$ to $M_{p, \varphi_{2}}(w)$.

$$
\left\|M_{\Omega, b}(f)\right\|_{M_{p, \varphi_{2}}(w)} \lesssim\|f\|_{M_{p, \varphi_{1}}(w)} .
$$

Proof. When $q^{\prime} \leq p<\infty, w \in A_{p / q^{\prime}}$, by Lemma 5.3 and Theorem 2.3 with $\nu_{2}(r)=$ $\varphi_{2}(x, r)^{-1}, \nu_{1}(r)=\varphi_{1}(x, r)^{-1} w(B(x, r))^{-\frac{1}{p}}, g(r)=\|f\|_{L_{p, w}(B(x, r))}$ and $w(r)=w(B(x, r))^{-\frac{1}{p}} r^{-1}$ we have

$$
\begin{aligned}
& \left\|M_{\Omega, b}(f)\right\|_{M_{p, \varphi_{2}}(w)}=\sup _{x \in \mathbb{R}^{n}, r>0} \varphi_{2}(x, r)^{-1} w(B(x, r))^{-\frac{1}{p}}\left\|\mu_{\Omega, b}(f)\right\|_{L_{p, w}(B(x, r))} \\
& \lesssim\|b\|_{*} \sup _{x \in \mathbb{R}^{n}, r>0} \varphi_{2}(x, r)^{-1} \int_{r}^{\infty}\left(1+\ln \frac{t}{r}\right)\|f\|_{L_{p, w}(B(x, t))} w(B(x, t))^{-\frac{1}{p}} \frac{d t}{t} \\
& \lesssim\|b\|_{*} \sup _{x \in \mathbb{R}^{n}, r>0} \varphi_{1}(x, r)^{-1} w(B(x, r))^{-\frac{1}{p}}\|f\|_{L_{p, w}(B(x, r))} \\
& =\|b\|_{*}\|f\|_{M_{p, \varphi_{1}}(w)} .
\end{aligned}
$$

For the case of $1<p \leq q, w^{1-p^{\prime}} \in A_{p^{\prime} / q^{\prime}}$, by Lemma 4.1 and Theorem 2.3 with

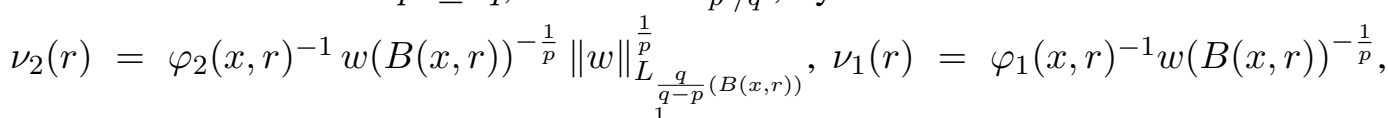
$g(r)=\|f\|_{L_{p, w}(B(x, r))}$ and $w(r)=\|w\|_{L_{\frac{q}{q-p}(B(x, r))}^{-\frac{1}{p}}}^{-\frac{1-p}{(B(x, r))}} r^{-1}$ we have

$$
\begin{aligned}
& \left\|M_{\Omega, b}(f)\right\|_{M_{p, \varphi_{2}}(w)}=\sup _{x \in \mathbb{R}^{n}, r>0} \varphi_{2}(x, r)^{-1} w(B(x, r))^{-\frac{1}{p}}\left\|\mu_{\Omega}(f)\right\|_{L_{p, w}(B(x, r))} \\
& \lesssim \sup _{x \in \mathbb{R}^{n}, r>0} \varphi_{2}(x, r)^{-1} w(B(x, r))^{-\frac{1}{p}}\|w\|_{L_{\frac{q}{q-p}(B)}^{\frac{1}{p}}}^{\infty}\left(1+\ln \frac{t}{r}\right)\|f\|_{L_{p, w}(B(x, t))}\|w\|_{L_{\frac{q}{q-p}(B(x, t))}^{-\frac{1}{p}}} \frac{d t}{t} \\
& \times \int_{r}^{\infty}\left(\sup _{x \in \mathbb{R}^{n}, r>0} \varphi_{1}(x, r)^{-1} w(B(x, r))^{-\frac{1}{p}}\|f\|_{L_{p, w}(B(x, r))}=\|f\|_{M_{p, \varphi_{1}}(w)} .\right.
\end{aligned}
$$

Acknowledgements The author thanks the referee(s) for careful reading the paper and useful comments. 


\section{References}

1. Agcayazi, M., Gogatishvili, A., Koca, K., Mustafayev, R.: A note on maximal commutators and commutators of maximal functions, J. Math. Soc. Japan. 67 (2), 581-593 (2015).

2. Akbulut, A., Guliyev, V.S., Mustafayev R.: On the boundedness of the maximal operator and singular integral operators in generalized Morrey spaces, Math. Bohem. 137 (1), 27-43 (2012).

3. Akbulut, A., Hamzayev, V.H., Safarov, Z.V.: Rough fractional multilinear integral operators on generalized weighted Morrey spaces, Azerb. J. Math. 6 (2), 128-142 (2016).

4. Alvarez, J., Bagby, R.J., Kurtz, D.S., Pérez C.: Weighted estimates for commutators of linear operators, Studia Math. 104, 195-209, (1993).

5. Burenkov, V., Gogatishvili, A., Guliyev, V., Mustafayev, R.: Boundedness of the fractional maximal operator in local Morrey-type spaces, Complex Var. Elliptic Equ. 55 (8-10), 739-758 (2010).

6. Calderon, A.P.: Commutators of singular integral operators, Proc. Natl. Acad. Sci. USA 53, 1092-1099 (1965).

7. Calderon, A.P.: Cauchy integrals on Lipschitz curves and related operators, Proc. Natl. Acad. Sci. USA 74 (4), 1324-1327 (1977).

8. Coifman, R., Rochberg, R., Weiss G.: Factorization theorems for Hardy spaces in several variables, Ann. of Math. 103 (2), 611-635 (1976).

9. Di Fazio, G., Ragusa, M.A.: Interior estimates in Morrey spaces for strong solutions to nondivergence form equations with discontinuous coefficients, J. Funct. Anal. 112, 241-256 (1993).

10. Fan, D., Lu, S., Yang, D.: Boundedness of operators in Morrey spaces on homogeneous spaces and its applications, Acta Math. Sinica (N. S.) 14, 625-634 (1998).

11. Eroglu, A., Omarova, M.N., Muradova, Sh.A.: Elliptic equations with measurable coefficients in generalized weighted Morrey spaces, Proc. Inst. Math. Mech. Natl. Acad. Sci. Azerb. 43 (2), 197-213 (2017).

12. Eroglu, A., Azizov, J.V.: A note on the fractional integral operators in generalized Morrey spaces on the Heisenberg group, Trans. Natl. Acad. Sci. Azerb. Ser. Phys.-Tech. Math. Sci. 37 (1), Mathematics, 86-91 (2017).

13. Deringoz, F.: Parametric Marcinkiewicz integral operator and its higher order commutators on generalized weighted Morrey spaces, Trans. Natl. Acad. Sci. Azerb. Ser. Phys.-Tech. Math. Sci. 37 (4), Mathematics, 24-32 (2017).

14. Duoandikoetxea, J.: Weighted norm inequalities for homogeneous singular integrals, Trans. Amer. Math. Soc. 336, 869-880 (1993).

15. Gadjiev, T., Galandarova, Sh., Guliyev, V.: Regularity in generalized Morrey spaces of solutions to higher order nondivergence elliptic equations with VMO coefficients, Electron. J. Qual. Theory Differ. Equ. Paper No. 55, 17 pp (2019).

16. Giaquinta, M.: Multiple integrals in the calculus of variations and nonlinear elliptic systems, Princeton Univ. Press, Princeton, NJ (1983).

17. Guliyev, V.S.: Integral operators on function spaces on the homogeneous groups and on domains in $\mathbb{R}^{n}$, Doctoral dissertation, (in Russian), Moscow, Mat. Inst. Steklov, 329 pp., (1994).

18. Guliyev, V.S.: Function spaces, integral operators and two weighted inequalities on homogeneous groups. Some applications, Baku. 1999, 1-332. (Russian)

19. Guliyev, V.S.: Boundedness of the maximal, potential and singular operators in the generalized Morrey spaces, J. Inequal. Appl. Art. ID 503948, 20 pp (2009).

20. Guliyev, V.S.: Generalized weighted Morrey spaces and higher order commutators of sublinear operators, Eurasian Math. J. 3 (3), 33-61 (2012). 
21. Guliyev, V.S.: Local generalized Morrey spaces and singular integrals with rough kernel, Azerb. J. Math. 3 (2), 79-94 (2013).

22. Guliyev, V.S., Karaman, T., Mustafayev, R.Ch., Serbetci, A.: Commutators of sublinear operators generated by Calderón-Zygmund operator on generalized weighted Morrey spaces, Czechoslovak Math. J. 64 (139) (2), 365-386 (2014).

23. Guliyev, V.S., Balakishiyev, A.S.: Parabolic fractional maximal and integral operators with rough kernels in parabolic generalized Morrey spaces, J. Math. Inequal. 9 (1), 257-276 (2015).

24. Guliyev, V., Gadjiev, T., Galandarova, Sh.: Dirichlet boundary value problems for uniformly elliptic equations in modified local generalized Sobolev-Morrey spaces, Electron. J. Qual. Theory Differ. Equ. Paper No. 71, 17 pp., (2017).

25. Guliyev, V.S., Ahmadli A.A., Omarova M.N., Softova L.: Global regularity in OrliczMorrey spaces of solutions to nondivergence elliptic equations with VMO coefficients, Electron. J. Differential Equations Paper No. 110, 24 pp., (2018).

26. Guliyev, V.S., Omarova, M.N., Softova, L.: The Dirichlet problem in a class of generalized weighted Morrey spaces, Proc. Inst. Math. Mech. Natl. Acad. Sci. Azerb. 45 (2), 270-285 (2019).

27. Janson, S.: On functions with conditions on the mean oscillation, Ark. Mat. 14 (2), 189-196 (1976).

28. Hamzayev, V.H.: Sublinear operators with rough kernel generated by CalderonZygmund operators and their commutators on generalized weighted Morrey spaces, Trans. Natl. Acad. Sci. Azerb. Ser. Phys.-Tech. Math. Sci. 38 (1), Mathematics, 79-94 (2018).

29. Ismayilova, A.F.: Commutators of the Marcinkiewicz integral on generalized weighted Morrey spaces, Trans. Natl. Acad. Sci. Azerb. Ser. Phys.-Tech. Math. Sci. 38 (4), Mathematics, 79-92 (2018).

30. Ismayilova, A.F.: Fractional maximal operator and its commutators on generalized weighted Morrey spaces, Trans. Natl. Acad. Sci. Azerb. Ser. Phys.-Tech. Math. Sci. 39 (4), Mathematics, 84-95 (2019).

31. Komori, Y., Shirai, S.: Weighted Morrey spaces and a singular integral operator, Math. Nachr. 282 (2), 219-231 (2009).

32. Kufner, A., John, O., Fuçik S.: Function Spaces, Noordhoff International Publishing: Leyden, Publishing House Czechoslovak Academy of Sciences: Prague (1977).

33. Mizuhara, T.: Boundedness of some classical operators on generalized Morrey spaces, Harmonic Analysis (S. Igari, Editor), ICM 90 Satellite Proceedings, Springer - Verlag, Tokyo, 183-189 (1991).

34. Morrey, C.B.: On the solutions of quasi-linear elliptic partial differential equations, Trans. Amer. Math. Soc. 43, 126-166 (1938).

35. Muckenhoupt, B.: Weighted norm inequalities for the Hardy maximal function, Trans. Amer. Math. Soc. 165, 207-226 (1972).

36. Muckenhoupt, B., Wheeden, R.: Weighted norm inequalities for fractional integrals, Trans. Amer. Math. Soc. 192, 261-274 (1974).

37. Muradova, Sh.A., Hamzayev, V.H.: Anisotropic maximal and singular integral operators in anisotropic generalized Morrey spaces, Trans. Natl. Acad. Sci. Azerb. Ser. Phys.-Tech. Math. Sci. 34 (4), Mathematics and Mechanics, 73-84 (2014).

38. Nakai, E.: Hardy-Littlewood maximal operator, singular integral operators and Riesz potentials on generalized Morrey spaces, Math. Nachr. 166, 95-103 (1994).

39. Sawano, Y.: A thought on generalized Morrey spaces, J. Indonesian Math. Soc. 25 (3), 210-281 (2019).

40. Watson, D.: Weighted estimates for singular integrals via Fourier transform estimates, Duke Math. J. 60, 389-399 (1990). 